\title{
Does Estradiol Orally and Vaginally Administered Together Impact Live Birth and Neonatal Outcome in Artificial Frozen-thawed Embryo Transfer Cycles: A Retrospective Cohort Study
}

\section{Yuan Liu}

Shanghai General Hospital, Shanghai Jiaotong University School of Medicine

\section{Yixia Yang}

Shanghai General Hospital, Shanghai Jiaotong University School of Medicine

\section{Xinting Zhou}

Shanghai General Hospital, Shanghai Jiaotong University School of Medicine

\section{Yanmei Hu}

Shanghai General Hospital, Shanghai Jiaotong University School of Medicine

YuWu ( $\nabla$ wuyu1970@yahoo.com )

Reproductive Medicine Center, Department of Obstetrics and Gynecology, Shanghai General Hospital, Shanghai Jiaotong University School of Medicine https://orcid.org/0000-0001-7808-8725

\section{Research article}

Keywords: FET, HRT, Estrogen Vaginally, Live Birth, Birthweight

Posted Date: July 20th, 2020

DOI: https://doi.org/10.21203/rs.3.rs-42796/v1

License: (c) (i) This work is licensed under a Creative Commons Attribution 4.0 International License.

Read Full License 


\section{Abstract}

Background: Previous studies have demonstrated that newborns from fresh embryo transfer are with higher risk of small for gestation (SGA) rate than those from frozen-thawed embryo transfer (FET). It is suggested that supraphysiologic serum estradiol in controlled ovarian stimulation (COS)is one of reasons. Out study aims to investigate whether exogenous estradiol delivered regimens have an impact on live birth rate and singleton birthweight in hormone replacement (HRT)-FET cycles.

Methods:This retrospective study involved patients undergoing their first FET with HRT endometrium preparation followed by two cleavage-staged embryos transfer, comparing orally and vaginal estradiol tablets (OVE) group versus oral estradiol tablets (OE) group from January 2015 to December 2018 at our center. A total of 792 patients fulfilled the criteria, including 282 live birth singletons. Live birth was the primary outcome. Secondary outcome included clinical pregnancy rate, singleton birthweight, large for gestational age (LGA) rate, SGA rate, preterm delivery rate.

Results:Patients in OVE group achieved higher serum estradiol level with more days of estradiol treatment. No difference in live birth (Adjusted OR 1.327; 95\% $\mathrm{Cl} 0.982,1.794, \mathrm{p}=0.066$ ) and clinical pregnancy rate (Adjusted OR 1.278; 95\% Cl 0.937, 1.743, $\mathrm{p}=0.121$ ) was found between OVE and OE groups. Estradiol route did not affect birth weight $(\beta=-30.962, S E=68.723, p=0.653)$, the odds of LGA (Adjusted OR 1.165; 95\% Cl 0.545, 2.490, $\mathrm{p}=0.694$ ), the odds of SGA (Adjusted OR 0.569; $95 \% \mathrm{Cl} 0.096$, $3.369, p=0.535$ ) or the preterm delivery rate (Adjusted OR 0.969; $95 \% \mathrm{Cl} 0.292,3.214, p=0.959$ ).

Conclusion:Estrogen orally and vaginally together did not have an impact on clinical outcomes and singleton birthweight compared to estrogen orally taken, but was accompanied with relative higher serum E2 level and potential maternal undesirable risks.

\section{Background}

Vitrification technology has played an instrumental role in the implementation of fertility preservation, single embryo transfer and freeze-all cycles and helped avoid ovarian hyperstimulation syndrome (OHSS) in assisted reproductive technology (ART). During frozen-thawed embryo transfer (FET), though no endometrial preparation protocol superior to others is found, hormone replacement treatment (HRT) is preferred by many centers due to the controlled exposure to exogenous steroids and adjustment of the timing of transfer. In HRT endometrial prepared FET cycles, estradiol and progesterone are sequentially administered to synchronize the window of embryo implantation with embryo transfer. Endometrium proliferated and ovulation was hampered due to the continued estrogen administration during the initial estrogen-only phase. Daily progesterone started before the scheduled embryo transfer. Adequate estradiol priming induced endometrial cells proliferation and appropriate progesterone receptors to develop endometrial receptivity.

The growing number of FET has made researchers to examine various aspects of this treatment strategy. Epidemiologic data has indicated that FETs are associated with increased risk of large and post- 
date babies and adverse perinatal outcomes like pregnancy related hypertension, postpartum hemorrhage, compared to fresh transfer[1-6]. The increased risk of preterm labor and small for gestational age (SGA) babies was found in fresh-transfer ART and was fundamentally decreased in FET. The reasons behind that varied. Though some reports suggested embryo cryopreservation altered epigenetics regulation inducing abnormal placentation and fetal growth, more studies advised the elevated level of estradiol which has been shown to impair trophoblastic invasion led to the smaller birthweight and shorter gestational weeks in fresh IVF cycles[7-12]. The negative effect of supraphysiologic E2 milieu on implantation and placentation was believed eliminated by the subsequent transfer of frozen-thawed embryos in the relatively steady endocrine environment. However, the duration and dose of estradiol administration in HRT-FET cycles intervened with the clinical and neonatal outcomes. Sekhon et al. have indicated that duration of estradiol supplementation before progesterone in artificial cycles did not impact frozen blastocyst transfer outcome, but inversely correlated with gestational age[13]. Constant dose or increasing dose of estrogen in HRT for endometrium preparation though didn't change live birth rate[14], but excessive duration of estradiol priming which was more than 28 days induced slacked pregnancy rate and increased miscarriage rates in FET[15].

In HRT endometrium preparation, different routes estrogen absorbed induced different serum estradiol level. Estrogen administered vaginally was firstly reported in 1988[16]. The alternative vaginal rings allowed a steady release of estrogen into the bloodstream. Estrogen administered vaginally seemed to cause higher circulating E2 level and even higher in endometrial tissue than estrogen administered orally[17]. The objective of the current retrospective study was to investigate whether estrogen orally and vaginally together administration made a difference to the live birth rate and singletons birthweight compared to estrogen orally administration. This model was chosen to specifically focus on the effect of vaginal absorbed estrogen induced higher E2 priming on FET clinical and neonatal outcomes.

\section{Methods}

Study design and patient population

This is a retrospective study which was undertaken at the assisted reproduction medicine department of Shanghai general hospital affiliated to Shanghai Jiao Tong university school of medicine. It involved 1005 women who had undergone their first FET from January 2015 to January 2018. Data was extracted from the database in our center.Patients inclusion criteria wasmaternal age $<48$, two Day 2 or Day 3 cleavage-staged embryos transfer following HRT endometrium preparation. The following patients were excluded from the analysis: (1) Patients with cryopreserved oocytes or donor oocytes. (2) Patients who had prior attempts at conception via IVF and FET. (3) Patients who were in HRT treatment with Sidenafil or growth hormone to improve endometrium development. (4) Patients with endometrium thickness less than $6 \mathrm{~mm}$ at endometrium transform day. (5) Patientswith endometrial polyps, submucosal myomas, or

endometrium separation. (6) Patients with estrogen administration beyond the doctor's order. (7) Patients with duration of estradiol administration $>28$ days. 792 women and 228 live birth singletons were finally 
included in the criteria (Figure 1). Institutional review board and ethics committee of Shanghai General Hospital approval was obtained (2020KY016).

IVF and Laboratory protocols

Ovarian stimulation, oocyte retrieval and fertilization procedures have been previously presented[18]. As for IVF, oocytes were inseminated with human tubal fluid with $10 \%$ serum substitute supplement and around 300000 progressively motile spermatozoa. As for ICSI, oocytes were laid in the fertilization medium immediately after microinjection. Fertilization was evaluated 18 hours post fertilization procedures. Embryos were cultured in early cleavage medium before Day3 and in multiblast medium afterwards. All embryos were cultured in incubator at $37^{\circ} \mathrm{C}$, under $6 \% \mathrm{CO}_{2}$ and $5 \% \mathrm{O}_{2}$. Embryo development was evaluated on Day2, Day3, Day5 and Day6. Day2 or Day3 cleavage-stage embryos with at least three or six blastomeres respectively and less than $20 \%$ fragmentation were eligible for cryopreservation. The criteria for good-quality embryo were: 4 to 5 cells without fragmentation for Day 2 embryos, 7 to 9 cells with less than $20 \%$ fragmentation for Day3 embryos.

Frozen embryo transfer protocols

In a subsequent cycle, patients were administered estrogen and consecutive progesterone for endometrial preparation before FET. All patients started with estrogen administered orally (Estradiol Valerate or Estradiol Femoston) $6 \mathrm{mg}$ per day from the second day of menstruation and continued for one week. Subsequently, some patients continued the same regimen until progesterone was applied. "OE group" was used for these patients. Other patients were given extra estradiol supplement vaginally, Estradiol Femoston $2 \mathrm{mg}$ per day, combined with the oral usage for another7-21 days. "OVE group" was used for these patients (Figure 2). The whole estradiol administration duration could vary from 10 to 28 days. Patients came for visit every week. We performed transvaginal ultrasonagraphy every week to assess patients' endometrium. Vaginally administered estrogen was usually added if the endometrium thickness was less than $6 \mathrm{~mm}$ in patient's first visit. Additionally, it was also depended on physician's preference. Serum E2, LH and progesterone level were measured at each visit to detect premature ovulation. Once the timing of FET was determined, progesterone intramuscularly or Crinone vaginally was initiated daily. They both combined with dydrogesterone orally $40 \mathrm{mg}$ per day and estradiol orally $6 \mathrm{mg}$ per day. Intramuscular Progesteron $60 \mathrm{mg}$ per day or Vaginal Crinone $90 \mathrm{mg}$ per day was chosen according to patient preference. Patients who were undertaken two Day 2 or Day 3 cleavage-staged embryos transferred started the progesterone 2 or 3 days before FET, respectively. The vitrification and thawing procedures were previously described[19]. Embryo transfer was performed via the same flexible catheter (20G)and transabdominal ultrasonography guidance. After embryo transfer, same daily estrogenand progesterone administration continued until a negative pregnancy testat the 14 thday after transfer. If pregnancy was achieved, hormone administration continued until 12 weeks' gestation.

Outcome measures and definitions 
In order to evaluate the impact of estradiol delivered orally and vaginally together on clinical outcome, the primary outcome analyzed was live birth rate. Clinical pregnancy rate, newborn birthweight, LGA, SGA, preterm delivery rate were secondary outcomes. Live birth was defined as a delivery of a viable infant after 28th gestational weeks. Clinical pregnancy was a pregnancy confirmed by the gestational sac or heart beat under sonography. Gestational age was calculated from 14 days before the embryo transfer. Preterm birth was defined as delivery between 32 to 37 gestational weeks. Small for gestational age (SGA) and large for gestational age (LGA) were defined as birthweight $<10$ th and $>90$ th percentile, respectively. $Z$ score was administered to calculate birthweight adjusted for gestational age and newborn gender with the formula: $\mathrm{Z}$ score $=(\mathrm{c}-\mathrm{m}) / \mathrm{s}$, where $\mathrm{c}$ is the newborn birthweight, $\mathrm{m}$ is the mean birthweight for the same sex and same gestational age in the reference group and $s$ is the standard deviation of the reference group. The reference is the Chinese singletons newborns[20].

Statistical analysis

Patients and singletons live birth demographic baseline, cycle characteristics, clinical and neonatal outcomes were compared with Student's t-test, Wilcoxon rank sum tests, chi-square and Fisher's exact tests, as appropriate. Whether binary live birth and clinical pregnancy were affected by the regimen of estradiol was assessed by multivariable logistic regression adjusting for maternal age, BMI, whether duration of estradiol treatment $>21$ days, whether at least one good quality embryo was transfered, the route of progesterone administered, endometrium thickness at endometrium transform day. Multivariable logistic regression was applied to evaluate estradiol regimen impact on preterm delivery, LGA and SGA rate, adjusting for the major covariates mentioned above plus newborn gender. Multiple linear regression was applied to investigate the independent effect of estrogen regimen on newborn birthweight. Adjusted odds ratios $(\mathrm{OR})$ and $95 \%$ confidence intervals $(95 \% \mathrm{Cl})$ were reported. All analyses were conducted with spss statistics. $P$ value $<0.05$ was considered statistically significant.

\section{Results}

\section{Clinical outcome}

This analysis included 792 women and 228 live birth singletons with the following outcomes: $45.1 \%$ clinical pregnancy rate, $36.4 \%$ live birth rate. There were 324 patients who were estrogen administered orally and vaginally (OVE group), and 468 patients who were estrogen administered orally only (OE group). Baseline demographics and characteristics were compared between patients with different estrogen regimen (Table 1). Among the 792 women, it did not reveal any significant difference for maternal age, BMI, whether there was at least one good quality embryo transferred, progesterone route, E2 level at 14th day after transfer and $P$ level at endometrium transform day between two groups. There were more patients with estradiol treatment days over 21 days in OVE group than in OE group. Serum E2 level at endometrium transform day from OVE group was significantly higher than those from OE group. Endometrium thickness at transform day was smaller in OVE group than OE group, as the same result for the endometrium thickness at HCG day in controlled ovarian stimulation (COS). No significant difference 
of live birth rate (Crude OR $1.229,95 \mathrm{Cl} \% 0.917,1.649$ ) and clinical pregnancy rate (Crude OR $1.260,95 \% \mathrm{Cl}$ $0.948,1.675$ ) was found between OVE and OE group (Table 2). Controlling for maternal age, BMI, whether estradiol duration was longer than 21 days, whether there was at least one good quality embryo transferred, the route of progesterone administration, endometrium thickness at endometrium transform day, estradiol administered regimen did not modify the odds of achieving live birth (Adjusted OR 1.327, $95 \% \mathrm{Cl} 0.982,1.794, \mathrm{p}=0.066$ ) or clinical pregnancy (Adjusted OR 1.278, 95\% Cl 0.937, 1.743, $\mathrm{p}=0.121$ ) (Table 2). Endometrium thickness at endometrium transform day positively increased the clinical pregnancy (Adjusted 1.184, 95\% $\mathrm{Cl} 1.026,1.365, \mathrm{p}=0.021$ ) with no impact on live birth rate (Adjusted OR $1.119,95 \% \mathrm{Cl} 0.968,1.295, \mathrm{p}=0.130$ ) (Supplementary Figure 1\&2). Maternal age and at least one good quality embryo transferred were the independent factors that increased the live birth rate and clinical pregnancy rate (Supplementary Figure 1\&2).

Neonatal outcome

To further explore the estrogen regimen impact on birthweight and gestational age, a cohort of 228 live birth singletons from 792 patients was further investigated. The singletons were divided in two groups which were OVE group, and OE group. Baseline demographic and cycle characteristics were presented in Table 3. Comparison between the two groups did not reveal any significant difference for maternal age, $\mathrm{BMI}$, whether there was at least one good quality embryo transferred and progesterone route. OVE group from 228 singletons cohort had more percentage of patients with longer days of estradiol treatment, thinner endometrium before FET and higher serum E2 level at endometrium transform day than OE group, as the same result with the 792 cohort (Table 3).

Neonatal outcomes stratified by the regimen of estradiol administered were also presented in Table 3 . Preterm delivery rate, mean birthweight and Z-scores were not different across two groups (Table 3). Given that gestational age at delivery is an important determinant of newborn birthweight, a subgroup analysis was conducted stratified by term delivery. The analysis of gestational age between 32 to 36 weeks was not performed because the number in this category was too small. As shown in Table 3 , Newborn gender, mean birthweight, LGA rate and SGA rate for term singletons were not different across the two groups.

In multivariate analyses (Table 4), the risk of preterm delivery (Adjusted OR $0.969,95 \% \mathrm{Cl} 0.292,3.214$ ) and the risk of LGA (Adjusted OR 1.165, 95\% Cl 0.545, 2.490), SGA in term delivery (Adjusted OR 0.569, $95 \% \mathrm{Cl} 0.096,3.369$ ) were not significantly different between two groups after adjusting for maternal age, $\mathrm{BMI}$, transfer with at least one good quality embryo, whether estrogen administration more than 21 days, endometrium thickness at endometrium transform day, progesterone route and newborn gender. The multivariate analysis of LGA and SGA for gestational age between 32 to 36 weeks was not performed because the number in this category was too small. After correction for a number of potential confounders, the route of estradiol administered was not correlated with neonatal birthweight $(\beta=-30.962$, $\mathrm{SE}=68.723, \mathrm{p}=0.653)($ Table 5). 


\section{Discussion}

From our study, estrogen vaginally and orally regimen did not improve clinical pregnancy rate or live birth rate compared to estrogen orally regimen in HRT endometrial preparation FET cycles. Adding estrogen vaginally supplement did not change the newborn weight, SGA, LGA or the preterm delivery rate of the live birth singletons either.

Nowadays, a growing number of reports advised better IVF outcomes after freeze-all policy and elective embryo cryopreservation[21-23]. Some research has suggested COS has made a detrimental effect on endometrium receptivity $[24,25]$. They reported lower implantation rates in fresh transfer cycles compared to FET cycles due to the supraphysiologic estrogen level on embryo-endometrium asynchrony and impaired endometrial receptivity after ovarian stimulation[26]. Adverse perinatal outcomes such as low birth weight newborn in term singletons have been associated with fresh IVF-ET because of the hyperestrogenic milieu generated during COS in IVF[7-11]. Excessive E2 priming induced from development and recruitment of multiple follicles can affect gene expression pattern change like Grb10 and GATA3, and generate epigenetic alterations in developing embryo and fetus through alterations in DNA methylation, histone alteration and others[27-30]. It is also reported that immune environment changed due to the increase of natural killer cells in oocytes during stimulated cycles compared with natural cycles[31]. Ovarian stimulation would probably not benefit implantation, placentation and subsequent fetal growth. Since freeze-all policy and FET have been welcomed as global trend in recent years due to the endometrial synchrony and less perinatal morbidity, we must recognize the adverse perinatal outcomes of FET, like higher risk of macrosomia, perinatal mortality and pregnancy complications like preeclampsia[32-35]. There is evidence that FET singletons have higher mean birthweight than singletons born after fresh embryo transfer and natural pregnancy[1]. A trend toward higher neonatal death was found in FET group compared to fresh ET group in a RCT of 1508 PCOS patients [36]. FET is not perfect without risks.

Dramatically large-spanned levels of serum estrogen were found between ovarian stimulated cycles and artificial FET cycles. If the supraphysilogic E2 priming was one important reason for the low birth weight and shorter gestational weeks in stimulated cycles singletons, we wanted to explore the impact of different serum E2 levels induced by different routes of estrogen on artificial FET clinical and neonatal outcome. Estradiol valerate orally has the structure of $17 \beta$-estradiol, which possessed low bioavailability. Only very small part of ingested dose reaches the circulation intact. While the vaginally delivered $17 \beta$-estradiol exerts the local effect on endometrium after vaginal epithelium absorption, bypassing the liver metabolism and achieving higher bioavailability[17]. The regimen of estrogen administered usually varies depending on clinical variables like endometrium thickness, previous clinical records and physician preference. Considering the rise in FET cycles applied in recent years, it is essential to investigate whether the peripheral serum estrogen levels induced by estradiol absorbed regimens can impact endometrial receptivity and placentation. There is scarce publication available regarding the effect of estrogen administered vaginally route in FET cycles. To our knowledge, one retrospective study of 247 artificial FET cycles has suggested that vaginal estrogen regimen did not promote implantation 
and pregnancy rate compared to oral regimen. But they didn't investigate the live birth rate[37]. The other prospective study of 78artificial FET cycles showed vaginal estradiol administration improved endometrial proliferation by eliciting thicker endometrium, but it did not dig and analyze clinical outcomes[38]. Our present study has expanded this concept by providing the comprehensive wellcontrolled analysis, which evaluated estrogen administration route impact on both live birth rate and perinatal outcome.

Sekhon et al. suggested duration of estradiol supplementation before progesterone in artificial cycles did not impact frozen blastocyst transfer outcome[13]. This effect may extend beyond the route estradiol absorbed as we demonstrated that estrogen vaginally or orally administered did not impact clinical pregnancy rate and live birth rate in artificial FET cycles. Estrogen administered vaginally and orally together induced higher serum estrogen with longer duration of estrogen supplement, but ended up with thinner endometrium compared to orally only group. It is noteworthy that the patients in OVE group probably had inferior endometrium proliferation capability, considering their thinner endometrium both in $\mathrm{COS}$ and the first visit in artificial FET cycles. From our result, estrogen vaginally administered didn't improve endometrium thickness and neither increased the successful rate in FET, but was accompanied with the higher circulating estradiol level. The extremely high E2 level might adversely affect the clotting system homeostasis with higher incidence of thromboembolic events[39]. Nevertheless, the high serum E2 priming caused by estrogen vaginally and orally delivered seemed to not have an impact on singletons preterm delivery rate, LGA, and SGA rate.

Our study was not designed to identify the mechanisms underlying the results. The reason why we didn't see the lower birthweight and shorter gestational weeks induced by the higher peripheral serum estradiol in our cohort may be speculated that the serum estradiol level in our analysis is still not high enough to create the supraphysiologic estradiol milieu as in fresh IVF cycles. The mean serum peak estradiol value in COS varied from 10460-15362 pmol/L. Estradiol higher than that cutoff value proclaimed the high risk of preterm labor and SGA[7, 11]. The median value of serum estrogen level at endometrium transform day in our study was 6106 and $992 \mathrm{pmol} / \mathrm{L}$ in two groups respectively, which were both much closer to serum E2 level in natural cycle. Additionally, we didn't use estrogen vaginally gel insertion replaced of estrogen orally taken. In OVE group, we still continued the orally-taken estrogen valerate and subsequently added estrogen gel vaginally in case of vaginal malabsorption in some patients.

The present study has following strengths. The data was collected from one IVF center whichguaranteed the same lab equipment and associated operating people and sonographers. This ensured the practice consistency. Male neonates have been predicted to be higher-weighted than female neonates. To control for infant gender and gestational age bias, z score was calculated across different groups. We also accounted for progesterone regimen as potential confounder in multivariate analysis. Progestin has been suggested to directly advance vascular proliferation during placentation[40]. A supraphysiologic progestin exposure in HRT could initiate excessively deep placentation which would make a difference in infant birthweight and obstetrical consequences[41]. The progesterone supplementation route in this analysis included two regimens. Though different progesterone regimens 
seemed to induce comparable pregnancy rate[42], taking into consideration of progesterone and estrogen regimen simultaneously greatly benefitted in our model. We reviewed the database and restricted the analysis to strict criteria. We tried to minimize any effect associated with uterine factor infertility by excluding patients with endometrium thickness less than $6 \mathrm{~mm}$ at endometrium transform day and patients who have presented with endometrial polyps, submucosal myomas, or endometrium separation. We didn't include patients with estrogen administered more than 28 days in our study to minimize the effect of extended duration of estrogen, as E2 priming longer than 28 days showed a slacked pregnancy rate in FET[15]. We didn't include blastocyst embryo transfer into the whole cohort to mitigate the possibleimpact of prolonged in vitro embryo culture on subsequent newborn birthweight and gestational weeks[43, 44]. Only homogenous group of patients who achieved adequate endometrial thickness in their first FET cycles were included to guarantee the relative good-quality embryo transfer and exclude the recurrent FET failure cases, as poor-quality embryos were put forward to be associated with lower birthweight[45]. The long-term follow up of the cohort allowed to provide important neonatal outcomes.

Our study is limited by its retrospective design. Whether and when the estrogen vaginally was administered depended on both patients' endometrium thickness and physician's preference. Patients added with estrogen vaginally administered are likely to possess inferior capability of endometrium growth and inherent uterine defects that may predispose them to thinner endometrium. The selection bias was possible. We couldn't account for all possible cofounding variables. We couldn't follow up the patient obstetrics details including hypertensive disorders of pregnancy or placental abnormalities, which hampered us to assess the pregnancy risk factors associated to preterm delivery. An evaluation of other perinataloutcomes such as pre-eclampsia, placenta accrete, previa, abruption hypertensive would allow us a better understanding of high serum estrogen level's impact on placental angiogenesis in late trimester.

\section{Conclusion}

This single-center analysis provided some evidence of the estradiol regimen for women undergoing HRT endometrium preparation FET cycles. Relatively higher serum E2 level induced by estrogen orally and vaginally together did not increase successful IVF outcome or patients' risk of delivery premature and low birth weight infants compared to estrogen orally only administration. Estrogen vaginally delivered didn't improve endometrium thickness much and neither successful rate in FET, but induced higher serum E2 value with potential maternal undesirable risks. Based on our findings, upon safe and effectiveness consideration, estrogen route could be adjusted to patient preference without compromising FET outcome. Patients should be adequately informed the potential risks and patient's opinion should be taken into consideration in medical practice. Large randomized controlled prospective clinic trials with detailed obstetric follow-up are needed to verify our result and allow a better understanding of the estrogen impact on placental-vessel associated obstetric complications.

\section{Abbreviations}


OHSS: ovarian hyperstimulation syndrome

ART: assisted reproductive technology

HRT: hormone replacement therapy

FET: frozen-thawed embryo transfer

cos: controlled ovarian stimulation

IVF: in vitro fertilization

ICSI: intracytoplasmic sperm injection

LGA: large for gestational age

SGA: small for gestational age

95\%Cl: 95\% confidence intervals

OR: odds ratios

BMI: body mass index

\section{Declarations}

Funding: Not applicable.

Competing interests: Not applicable.

Availability of data and material: Data available from the corresponding writer if reasonably requested.

Ethics approval:Institutional review board and ethics committee of Shanghai General Hospital approval was obtained (2020KY016).

Consent to participate and publish: Informed consent was obtained from all individual participants included in this study. The participants have consented to the submission of this study.

\section{Authors' contributions}

1. Liu: Project development, data analysis and manuscript writing

2. Yang: Data analysis and manuscript writing

3. Zhou: Data collection

4. Hu: Data collection

5. Wu: Idea conceiving, project development and manuscript editing 


\section{References}

1. Maheshwari A, Pandey S, Amalraj Raja E, Shetty A, Hamilton M, Bhattacharya S: Is frozen embryo transfer better for mothers and babies? Can cumulative meta-analysis provide a definitive answer? Hum Reprod Update 2018, 24(1):35-58.

2. Pinborg A, Henningsen AA, Loft A, Malchau SS, Forman J, Andersen AN: Large baby syndrome in singletons born after frozen embryo transfer (FET): is it due to maternal factors or the cryotechnique? Human reproduction (Oxford, England) 2014, 29(3):618-627.

3. Sha T, Yin X, Cheng W, Massey IY: Pregnancy-related complications and perinatal outcomes resulting from transfer of cryopreserved versus fresh embryos in vitro fertilization: a meta-analysis. Fertility and sterility 2018, 109(2):330-342 e339.

4. Sites CK, Wilson D, Barsky M, Bernson D, Bernstein IM, Boulet S, Zhang Y: Embryo cryopreservation and preeclampsia risk. Fertility and sterility 2017, 108(5):784-790.

5. Ishihara O, Araki R, Kuwahara A, Itakura A, Saito H, Adamson GD: Impact of frozen-thawed singleblastocyst transfer on maternal and neonatal outcome: an analysis of 277,042 singleembryo transfer cycles from 2008 to 2010 in Japan. Fertility and sterility 2014, 101(1):128-133.

6. Zhang B, Wei D, Legro RS, Shi Y, Li J, Zhang L, Hong Y, Sun G, Zhang T, Li W et al: Obstetric complications after frozen versus fresh embryo transfer in women with polycystic ovary syndrome: results from a randomized trial. Fertility and sterility 2018, 109(2):324-329.

7. Pereira N, Elias RT, Christos PJ, Petrini AC, Hancock K, Lekovich JP, Rosenwaks Z: Supraphysiologic estradiol is an independent predictor of low birth weight in full-term singletons born after fresh embryo transfer. Hum Reprod 2017, 32(7):1410-1417.

8. Zhang W, Ma Y, Xiong $Y$, Xiao X, Chen S, Wang X: Supraphysiological serum oestradiol negatively affects birthweight in cryopreserved embryo transfers: a retrospective cohort study. Reprod Biomed Online 2019, 39(2):312-320.

9. Hu XL, Feng C, Lin XH, Zhong ZX, Zhu YM, Lv PP, Lv M, Meng Y, Zhang D, Lu XE et al: High maternal serum estradiol environment in the first trimester is associated with the increased risk of small-forgestational-age birth. J Clin Endocrinol Metab 2014, 99(6):2217-2224.

10. Liu S, Kuang Y, Wu Y, Feng Y, Lyu Q, Wang L, Sun Y, Sun X: High oestradiol concentration after ovarian stimulation is associated with lower maternal serum beta-HCG concentration and neonatal birth weight. Reprod Biomed Online 2017, 35(2):189-196.

11. Imudia AN, Awonuga AO, Doyle JO, Kaimal AJ, Wright DL, Toth TL, Styer AK: Peak serum estradiol level during controlled ovarian hyperstimulation is associated with increased risk of small for gestational age and preeclampsia in singleton pregnancies after in vitro fertilization. Fertil Steril 2012, 97(6):1374-1379.

12. Jarvela IY, Pelkonen S, Uimari O, Makikallio K, Puukka K, Ruokonen A, Tekay A, Martikainen H: Controlled ovarian hyperstimulation leads to high progesterone and estradiol levels during early pregnancy. Hum Reprod 2014, 29(11):2393-2401. 
13. Sekhon L, Feuerstein J, Pan S, Overbey J, Lee JA, Briton-Jones C, Flisser E, Stein DE, Mukherjee T, Grunfeld $L$ et al: Endometrial preparation before the transfer of single, vitrified-warmed, euploid blastocysts: does the duration of estradiol treatment influence clinical outcome? Fertil Steril 2019, 111(6):1177-1185.e1173.

14. Madero S, Rodriguez A, Vassena R, Vernaeve V: Endometrial preparation: effect of estrogen dose and administration route on reproductive outcomes in oocyte donation cycles with fresh embryo transfer. Hum Reprod 2016, 31(8):1755-1764.

15. Bourdon M, Santulli P, Kefelian F, Vienet-Legue L, Maignien C, Pocate-Cheriet K, de Mouzon J, Marcellin L, Chapron C: Prolonged estrogen (E2) treatment prior to frozen-blastocyst transfer decreases the live birth rate. Hum Reprod 2018, 33(5):905-913.

16. Rosenwaks Z, Navot D, Veeck L, Liu HC, Steingold K, Kreiner D, Droesch K, Stumpf P, Muasher SJ: Oocyte donation. The Norfolk Program. Ann N Y Acad Sci 1988, 541:728-741.

17. Paulson RJ: Hormonal induction of endometrial receptivity. Fertil Steril 2011, 96(3):530-535.

18. Zhang J, Liu H, Mao X, Chen Q, Si J, Fan Y, Xiao Y, Wang Y, Kuang Y: Effect of endometrial thickness on birthweight in frozen embryo transfer cycles: an analysis including 6181 singleton newborns. Human reproduction (Oxford, England) 2019, 34(9):1707-1715.

19. Rodriguez-Purata J, Lee J, Whitehouse M, Duke M, Grunfeld L, Sandler B, Copperman A, Mukherjee T: Reproductive outcome is optimized by genomic embryo screening, vitrification, and subsequent transfer into a prepared synchronous endometrium. J Assist Reprod Genet 2016, 33(3):401-412.

20. Dai L, Deng C, Li Y, Zhu J, Mu Y, Deng Y, Mao M, Wang Y, Li Q, Ma S et al: Birth weight reference percentiles for Chinese. PloS one 2014, 9(8):e104779.

21. Shapiro BS, Daneshmand ST, Garner FC, Aguirre M, Hudson C: Clinical rationale for cryopreservation of entire embryo cohorts in lieu of fresh transfer. Fertil Steril 2014, 102(1):3-9.

22. Shapiro BS, Daneshmand ST, Garner FC, Aguirre M, Hudson C, Thomas S: Evidence of impaired endometrial receptivity after ovarian stimulation for in vitro fertilization: a prospective randomized trial comparing fresh and frozen-thawed embryo transfers in high responders. Fertil Steril 2011, 96(2):516-518.

23. Shapiro BS, Daneshmand ST, Garner FC, Aguirre M, Hudson C, Thomas S: Evidence of impaired endometrial receptivity after ovarian stimulation for in vitro fertilization: a prospective randomized trial comparing fresh and frozen-thawed embryo transfer in normal responders. Fertil Steril 2011, 96(2):344-348.

24. Mirkin S, Nikas G, Hsiu JG, Diaz J, Oehninger S: Gene expression profiles and structural/functional features of the peri-implantation endometrium in natural and gonadotropin-stimulated cycles. $J$ Clin Endocrinol Metab 2004, 89(11):5742-5752.

25. Basir GS, O WS, Ng EH, Ho PC: Morphometric analysis of peri-implantation endometrium in patients having excessively high oestradiol concentrations after ovarian stimulation. Hum Reprod 2001, 16(3):435-440. 
26. Weinerman R, Mainigi M: Why we should transfer frozen instead of fresh embryos: the translational rationale. Fertil Steril 2014, 102(1):10-18.

27. Horcajadas JA, Riesewijk A, Polman J, van Os R, Pellicer A, Mosselman S, Simon C: Effect of controlled ovarian hyperstimulation in IVF on endometrial gene expression profiles. Mol Hum Reprod 2005, 11(3):195-205.

28. Horcajadas JA, Minguez P, Dopazo J, Esteban FJ, Dominguez F, Giudice LC, Pellicer A, Simon C: Controlled ovarian stimulation induces a functional genomic delay of the endometrium with potential clinical implications. J Clin Endocrinol Metab 2008, 93(11):4500-4510.

29. Haouzi D, Assou S, Mahmoud K, Tondeur S, Reme T, Hedon B, De Vos J, Hamamah S: Gene expression profile of human endometrial receptivity: comparison between natural and stimulated cycles for the same patients. Hum Reprod 2009, 24(6):1436-1445.

30. Lee B, Kroener LL, Xu N, Wang ET, Banks A, Williams J, 3rd, Goodarzi MO, Chen YI, Tang J, Wang Y et al: Function and Hormonal Regulation of GATA3 in Human First Trimester Placentation. Biol Reprod 2016, 95(5):113.

31. Junovich G, Mayer Y, Azpiroz A, Daher S, Iglesias A, Zylverstein C, Gentile T, Pasqualini S, Markert UR, Gutierrez G: Ovarian stimulation affects the levels of regulatory endometrial NK cells and angiogenic cytokine VEGF. Am J Reprod Immunol 2011, 65(2):146-153.

32. Wennerholm UB, Henningsen AK, Romundstad LB, Bergh C, Pinborg A, Skjaerven R, Forman J, Gissler $M$, Nygren KG, Tiitinen A: Perinatal outcomes of children born after frozen-thawed embryo transfer: a Nordic cohort study from the CoNARTaS group. Hum Reprod 2013, 28(9):2545-2553.

33. Shi Y, Sun Y, Hao C, Zhang H, Wei D, Zhang Y, Zhu Y, Deng X, Qi X, Li H et al: Transfer of Fresh versus Frozen Embryos in Ovulatory Women. N Engl J Med 2018, 378(2):126-136.

34. Sazonova A, Kallen K, Thurin-Kjellberg A, Wennerholm UB, Bergh C: Obstetric outcome in singletons after in vitro fertilization with cryopreserved/thawed embryos. Hum Reprod 2012, 27(5):1343-1350.

35. Opdahl S, Henningsen AA, Tiitinen A, Bergh C, Pinborg A, Romundstad PR, Wennerholm UB, Gissler M, Skjaerven R, Romundstad LB: Risk of hypertensive disorders in pregnancies following assisted reproductive technology: a cohort study from the CoNARTaS group. Hum Reprod 2015, 30(7):17241731.

36. Chen ZJ, Shi Y, Sun Y, Zhang B, Liang X, Cao Y, Yang J, Liu J, Wei D, Weng N et al: Fresh versus Frozen Embryos for Infertility in the Polycystic Ovary Syndrome. N Engl J Med 2016, 375(6):523-533.

37. Liao X, Li Z, Dong X, Zhang H: Comparison between oral and vaginal estrogen usage in inadequate endometrial patients for frozen-thawed blastocysts transfer. Int J Clin Exp Pathol 2014, 7(10):69926997.

38. Fanchin R, Righini C, Schonauer LM, Olivennes F, Cunha Filho JS, Frydman R: Vaginal versus oral $E(2)$ administration: effects on endometrial thickness, uterine perfusion, and contractility. Fertil Steril 2001, 76(5):994-998.

39. Magnusson A, Kallen K, Thurin-Kjellberg A, Bergh C: The number of oocytes retrieved during IVF: a balance between efficacy and safety. Human reproduction (Oxford, England) 2018, 33(1):58-64. 
40. Walter LM, Rogers PA, Girling JE: The role of progesterone in endometrial angiogenesis in pregnant and ovariectomised mice. Reproduction (Cambridge, England) 2005, 129(6):765-777.

41. Pirtea P, de Ziegler D, Ayoubi JM: Implantation rates of euploid embryos are not influenced by the duration of estradiol priming, but the hormonal environmentestradiol and progesterone-may affect placentation. Fertil Steril 2019, 111(6):1117-1118.

42. Shapiro DB, Pappadakis JA, Ellsworth NM, Hait HI, Nagy ZP: Progesterone replacement with vaginal gel versus i.m. injection: cycle and pregnancy outcomes in IVF patients receiving vitrified blastocysts. Human reproduction (Oxford, England) 2014, 29(8):1706-1711.

43. Zhang J, Wang Y, Liu H, Mao X, Chen Q, Fan Y, Xiao Y, Kuang Y: Effect of in vitro culture period on birth weight after vitrified-warmed transfer cycles: analysis of 4,201 singleton newborns. Fertil Steril 2019, 111(1):97-104.

44. Ginstrom Ernstad E, Spangmose AL, Opdahl S, Henningsen AA, Romundstad LB, Tiitinen A, Gissler M, Wennerholm UB, Pinborg A, Bergh $C$ et al: Perinatal and maternal outcome after vitrification of blastocysts: a Nordic study in singletons from the CoNARTaS group. Hum Reprod 2019, 34(11):22822289.

45. Zhang J, Huang J, Liu H, Wang B, Yang X, Shen X, Mao X, Wang Y, Kuang Y: The impact of embryo quality on singleton birthweight in vitrified-thawed single blastocyst transfer cycles. Human reproduction (Oxford, England) 2020, 35(2):308-316.

\section{Tables}

Table 1: Baseline demographic and cycle characteristics according to estrogen regimen 


\begin{tabular}{|c|c|c|c|}
\hline & $\begin{array}{l}\text { OVE group } \\
(\mathrm{N}=324)\end{array}$ & OE group $(\mathrm{N}=468)$ & $\mathbf{P}$ \\
\hline Maternal age $(y)$ & $30.87 \pm 4.51$ & $30.75 \pm 4.5$ & 0.717 \\
\hline BMI & $21.157 \pm 2.91$ & $21.57 \pm 3.2$ & 0.059 \\
\hline At least one good quality embryo & 282(87.04) & 407(86.97) & 0.977 \\
\hline \multicolumn{4}{|l|}{ Days of estradiol administration } \\
\hline$>21$ days & 35 & 5 & $<0.001$ \\
\hline$\leq 21$ days & 289 & 463 & \\
\hline $\begin{array}{l}\text { Endometrium thicknessat endometrium } \\
\text { transform day (mm) }\end{array}$ & $8.75(8.2,9.325)$ & $9(8.6,9.7)$ & $<0.001$ \\
\hline $\begin{array}{l}\text { Endometrium thickness at Hcg day in COS } \\
(\mathrm{mm})\end{array}$ & $\begin{array}{l}8.85(7.4,10) \\
(\mathrm{N}=306)\end{array}$ & $\begin{array}{l}9.8(7.8,12) \\
(\mathrm{N}=441)\end{array}$ & $<0.001$ \\
\hline \multicolumn{4}{|l|}{ Progesterone route } \\
\hline Intramuscular & 115 & 193 & 0.103 \\
\hline Vaginal & 209 & 275 & \\
\hline E2 level at endometrium transform day(pmol/L) & $\begin{array}{l}6105.5(2234 \\
9065.25) \\
(\mathrm{N}=292)\end{array}$ & $\begin{array}{l}992.5(644.5 \\
1400.75) \\
(\mathrm{N}=444)\end{array}$ & $<0.001$ \\
\hline E2 level at day 14 after transfer(pmol/L) & $\begin{array}{l}1346(969,1884) \\
(N=257)\end{array}$ & $\begin{array}{l}1420(1038,1875) \\
(\mathrm{N}=373)\end{array}$ & 0.364 \\
\hline P level at endometrium transform day(pmol/L) & $\begin{array}{l}1.11(0.67,1.66) \\
(\mathrm{N}=291)\end{array}$ & $\begin{array}{l}1.17(0.68,1.79) \\
(\mathrm{N}=442)\end{array}$ & 0.375 \\
\hline
\end{tabular}

Note: Data are presented as mean \pm SD for continuous variables in formal distribution, median (first quartile, third quartile) for continuous variables in informal distribution. $P$ values were assessed with the use of $t$ tests or Wilcoxon rank sum tests or chi-square. OVE group stands for estradiol orally and vaginally administered together group. OE group stands for estradiol orally administered group. COS stands for controlled ovarian stimulation.

Table 2₫Clinical outcomes according to different estrogen route 


\begin{tabular}{|c|c|c|c|c|}
\hline & $\begin{array}{l}\text { OVE group } \\
(\mathrm{N}=324)\end{array}$ & $\begin{array}{l}\text { OE group } \\
(n=468)\end{array}$ & Crude OR(95\%Cl) & $\begin{array}{l}\text { Adjusted } \\
\text { OR(95\% } 9 \text { Cl) }\end{array}$ \\
\hline $\begin{array}{l}\text { Clinical } \\
\text { pregnancy }\end{array}$ & $157 \rrbracket 48.46 \rrbracket$ & $200 \otimes 42.74 \rrbracket$ & $\begin{array}{l}1.260(0.948- \\
1.675)\end{array}$ & $\begin{array}{l}1.278(0.937- \\
1.743)\end{array}$ \\
\hline Live birth & $127 \rrbracket 39.2 \rrbracket$ & 161®34.4区 & $\begin{array}{l}1.229(0.917- \\
1.649)\end{array}$ & $\begin{array}{l}1.327(0.982- \\
1.794)\end{array}$ \\
\hline
\end{tabular}

Note: Analyses were adjusted for maternal age, BMI, whether days of estrogen treatment> 21 , whether at least one good quality embryo was transferred, endometrium thickness at endometrium transform day, progesterone regimen.OE group stands for estradiol orally administered group. COS stands for controlled ovarian stimulation. $\mathrm{Cl}=$ confidence interval; $\mathrm{OR}=$ odds ratio.

Table 3aBaseline demographics, cycle characteristics and neonatal outcomes of singletons live birth according to different estradiol route 


\begin{tabular}{|c|c|c|c|}
\hline & $\begin{array}{l}\text { Vaginal and oral } \\
(\mathrm{N}=98)\end{array}$ & Oral only $(\mathrm{N}=130)$ & $\mathbf{P}$ \\
\hline Maternal age $(y)$ & $29.96 \pm 3.83$ & $30.58 \pm 3.95$ & 0.236 \\
\hline BMI & $21.3 \pm 2.91$ & $21.48 \pm 3.12$ & 0.660 \\
\hline At least one good quality embryo & 90 & 122 & 0.923 \\
\hline $\begin{array}{l}\text { Endometrium thickness at endometrium } \\
\text { transform day }(\mathrm{mm})\end{array}$ & $8.8(8.2,9.4)$ & $9.0(8.6,9.9)$ & 0.004 \\
\hline $\begin{array}{l}\text { Endometrium thickness at hcg day in cos } \\
(\mathrm{mm})\end{array}$ & $9(8,10)$ & $9.75(6.75,11.7)$ & 0.100 \\
\hline \multicolumn{4}{|l|}{ Days of estradiol administration } \\
\hline$>21$ days & 12 & 1 & 0.001 \\
\hline$\leq 21$ days & 86 & 129 & \\
\hline \multirow{2}{*}{$\begin{array}{l}\text { E2 level at endometrium transform } \\
\text { day(pmol/L) }\end{array}$} & $5977(1882,9043)$ & $962(647.75,1364.5)$ & $<0.001$ \\
\hline & $(\mathrm{N}=89)$ & $(N=120)$ & \\
\hline \multicolumn{4}{|l|}{ Progesterone route } \\
\hline Intramuscular & 34 & 57 & 0.162 \\
\hline Vaginal & 64 & 73 & \\
\hline Male & 51 & 67 & \\
\hline \multicolumn{4}{|l|}{ Gestational Age } \\
\hline $32-36$ & 5 & 8 & 0.735 \\
\hline$>37$ & 93 & 122 & \\
\hline Birth weight & $3222 \pm 215.43$ & $3209.67 \pm 228.09$ & 0.679 \\
\hline Z score & $0.35 \pm 0.89$ & $0.38 \pm 1.12$ & 0.793 \\
\hline Gestational age>37weeks(n) & 93 & 122 & 0.735 \\
\hline \multicolumn{4}{|l|}{ New born gender } \\
\hline Female & 44 & 60 & 0.786 \\
\hline Male & 49 & 62 & \\
\hline Birthweight(g) & $3411.72 \pm 379.45$ & $3415.06 \pm 461.54$ & 0.954 \\
\hline Small for gestational age (SGA) & 2 & 5 & 0.693 \\
\hline Large for gestational age (LGA) & 18 & 21 & 0.660 \\
\hline
\end{tabular}


quartile, third quartile) for continuous variables in informal distribution. P values were assessed with the use of $t$ tests or Wilcoxon rank sum tests or chi-square (Fisher's exact tests as appropriate). COS stands for controlled ovarian stimulation. SGA stands for small for gestational age. LGA stands for large for gestational age.

Table 4: Crude and adjusted ORs of birthweight categories in singleton births.

\begin{tabular}{|lll|}
\hline & Crude OR (95\%Cl) & Adjusted OR (95\%Cl) \\
\hline Preterm delivery & $1.220(0.386-3.850)$ & $0.969(0.292-3.214)$ \\
\hline Gestational age $>37$ weeks & & \\
\hline LGA & $1.168(0.584-2.334)$ & $1.165(0.545-2.490)$ \\
\hline SGA & $0.521(0.099-2.743)$ & $0.569(0.096-3.369)$ \\
\hline
\end{tabular}

Note: Analyses were adjusted for maternal age, BMI, transfer with at least one good quality embryo, endometrium thickness, whether estrogen administration $>21$ days, progesterone route, newborn gender. $\mathrm{Cl}=$ confidence interval; $\mathrm{OR}=$ odds ratio.SGA stands for small for gestational age. LGA stands for large for gestational age.

\section{Figures}


Included: 1005 persons

Age $<48$ years

2 Day2 or Day3 embryos transferred First FET

\begin{tabular}{|l|l|}
\hline Excluded: \\
Donor oocytes \\
Prior attempts of conception via IVF or FET \\
Sidenafil or growth hormone \\
Endometrium thickness $<6 \mathrm{~mm}$ at endometrium transfer day \\
Endometrial polyps, submucosal myomas,or endometrium \\
separation \\
Beyond doctor's order \\
Estradiol administration $>28$ days
\end{tabular}

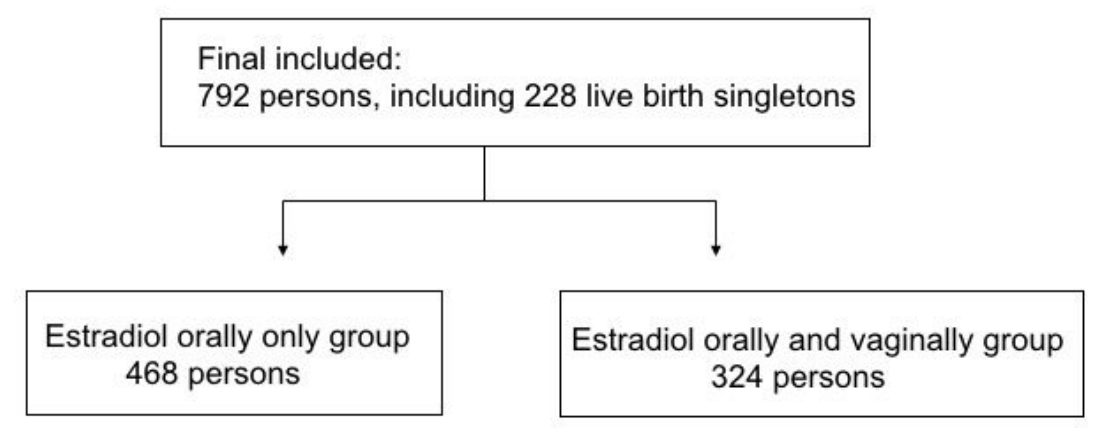

Figure 1

Flow diagram of patient inclusion.
OE group

\begin{tabular}{|c|c|}
\hline Estrogen orally & Estrogen orally \\
\hline 7 days & 3-21 days \\
\hline
\end{tabular}
Endometrium
transform day
Progesterone+Estrogen orally
Endometrium transform day
OVE group
Estrogen orally Estrogen orally + vaginally

Figure 2

Estrogen priming flow.

\section{Supplementary Files}


This is a list of supplementary files associated with this preprint. Click to download.

- SupplementalFigure2.jpg

- SupplementalFigure1.jpg 